\title{
Rheological Study of Dextran-Modified Magnetite Nanoparticle Water Suspension
}

\author{
A. Józefczak · T. Hornowski · Z. Rozynek • \\ A. Skumiel • J. O. Fossum
}

Received: 15 April 2012 / Accepted: 4 April 2013 / Published online: 24 April 2013

(C) The Author(s) 2013. This article is published with open access at Springerlink.com

\begin{abstract}
The aim of this work is to investigate the effect of surface modification of superparamagnetic magnetite nanoparticles (sterically stabilized by sodium oleate) by the dextran biocompatible layer on the rheological behavior of water-based magnetic fluids. The flow curves were measured as a function of the magnetic field strength by means of rheometry. The measured viscosity is generally dependent on both the particle concentration and the geometrical factors such as the particle shape and thickness of the adsorbed layers. The rheological properties of the magnetic fluids studied show the effect of the magnetic field strength and the presence of the surfactant second layer (dextran) on their viscosity.
\end{abstract}

Keywords Fluid flow $\cdot$ Magnetic fluid $\cdot$ Nanoparticles $\cdot$ Rheological properties

\section{Introduction}

Iron oxide nanoparticles are being developed for a wide range of biomedicine applications and diagnostics (magnetic drug targeting, magnetic hyperthermia, MRI contrast

\footnotetext{
A. Józefczak $(\varangle) \cdot$ T. Hornowski · A. Skumiel Institute of Acoustics, Faculty of Physics, Adam Mickiewicz University, Umultowska 85, 61-614 Poznań, Poland

e-mail: aras@amu.edu.pl

Z. Rozynek · J. O. Fossum

Department of Physics, Norwegian University of Science and Technology (NTNU), Høgskoleringen 5, 7491 Trondheim, Norway

J. O. Fossum

Center for Advanced Study (CAS) at the Norwegian Academy of Science and Letters, Drammensveien 78, 0271 Oslo, Norway
} 
agent). The delivery of nanoparticles to a human body usually requires suspending the particles in a water-based fluid. A colloidal suspension of single magnetic domain particles is named a magnetic fluid (MF) [1]. The stability and biocompatibility of the colloid suspensions are greatly improved by the addition of suitable polymeric dispersants [2]. Thus, nanoparticles become composite objects made of a magnetic core surrounded by one or more nonmagnetic shells. Polysaccharides, due to their unique physicochemical properties and excellent biocompatibility, should be appropriate to prepare nanoparticles applicable for biomedical purposes. Dextran, one kind of watersoluble polysaccharide, is produced industrially on a large scale and has found wide application in food and medical fields for more than half a century [3]. From a biomedical point of view, the adsorbed polymer layer should completely cover the particles and be as dense as possible in order to protect the iron oxide core against contact with blood proteins and phagocytosis-associated receptors [2].

For biomedical applications it is necessary to investigate the rheological behavior of a nanoparticle suspension. This report describes dextran surface modification of superparamagnetic magnetite nanoparticles (sterically stabilized by sodium oleate) prepared by the co-precipitation method. The aim of this work is to investigate the effect of biocompatible layers (dextran) on the rheological behavior of magnetic fluids. The viscosity measurement is a useful method of characterizing the particles in such a suspension. It is not only the particle concentration that contributes to the viscosity, but also the particle charge and shape, and also the dimensions of any adsorbed layers [4]. The rheological properties of a magnetic fluid are correlative to their microstructures.

In addition, the magnetic-field-dependent rheological properties of nanoparticle suspensions were studied. The experimentally observed phase condensation belongs to the most important structuring phenomena in magnetic nanocolloids [5-7]. As a result of the magnetically induced phase condensation, the magnetic nanocolloid separates into two coexisting phases. The condensed phase droplets containing highly packed magnetic nanoparticles are suspended in the matrix of the diluted phase. In the presence of an external magnetic field, the condensed phase drops are prolate spheroids aligned in the field direction. The colloidal stability of magnetic nanofluids with respect to the phase separation phenomenon depends on a variety of factors such as the magnetic nanoparticle concentration, the size and size polydispersity, the colloidal stabilization efficiency, the temperature, and the magnetic field intensity [5]. The formation of drop-like clusters leads to the appearance of viscoelastic effects or other non-Newtonian features in the magnetic nanoparticle suspension in the presence of a magnetic field $[8,9]$.

\section{Sample Preparation and Magnetic Properties}

The superparamagnetic nanoparticles were prepared by co-precipitation using ferric and ferrous salts and ammonium hydroxide. The freshly prepared magnetic nanoparticles (magnetite) were sterically stabilized by sodium oleate (ca. 1:1 to $\mathrm{Fe}_{3} \mathrm{O}_{4}$ ) to prevent their agglomeration. By centrifuging $30 \mathrm{~min}$ at $9000 \mathrm{rpm}$, an initial magnetic fluid (sample 1-MF, Fig. 1a) arose. Then, dextran (average molecular weight $=64000$ Daltons) was added to achieve a dextran-to- $\mathrm{Fe}_{3} \mathrm{O}_{4}$ mass ratio equal to 0.5 
Fig. 1 Structures of magnetic nanoparticles: (a) direct coating of magnetic core with sodium oleate shell and (b) direct coating of magnetic core with sodium oleate shell and biocompatible layers-dextran (a)

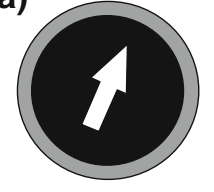

(b)

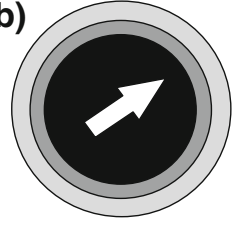

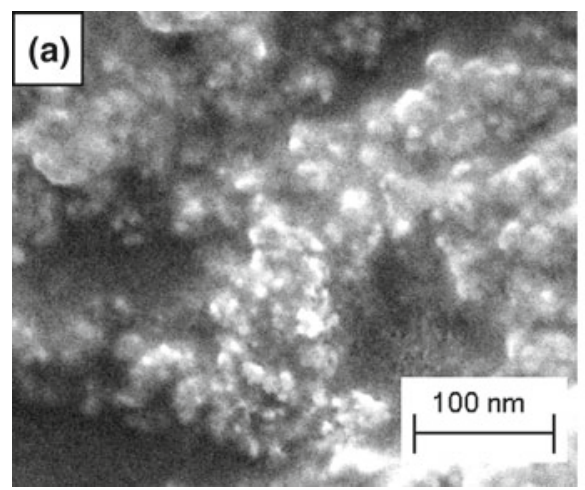

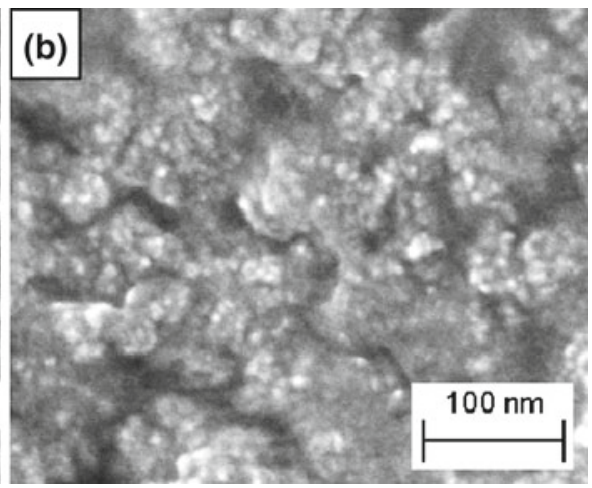

Fig. 2 SEM image of (a) MF and (b) MF dextran

(sample 2-MF dextran, Fig. 1b) [10]. Dextran is a polymer $\left(\mathrm{C}_{6} \mathrm{H}_{10} \mathrm{O}_{5}\right)_{n}$, of anhydroglucose having mainly alpha-D(1-6) linkages with some unusual 1,3 glucosidic linkages at branching points [2].

The morphology of the prepared nanoparticles was observed by scanning electron microscopy (SEM). A field emission scanning electron microscope (Model S-4300SE from Hitachi placed at NTNU) was used in these studies. SEM samples were prepared by placing a drop of the as-prepared suspension on a grid and then drying it in a desiccator. Figure 2 shows the SEM images of nanoparticles and dextran-modified magnetite nanoparticles. SEM analysis of the MF and MF dextran samples showed almost spherical shape of particles.

Our ultrasonic studies show that the surface modification of magnetic nanoparticles with dextran enhances the stability of biocompatible magnetic fluids [11].

The magnetic properties were measured at $290 \mathrm{~K}$ using a SQUID magnetometer. Figure 3 shows the magnetization curves from which the volume concentration of magnetite particles in magnetic liquids, the average diameter of magnetite particles (Table 1), and the particle magnetic core size distribution function (PSD) (Fig. 4) were determined. The description of the method used to calculate the PSD and its parameters from magnetic measurements can be found in $[12,13]$. It can be seen from Fig. 4 that both samples have approximately the same magnetic properties (concentration of magnetic material, $\phi_{\mathrm{m}}=1.4$ ) and magnetic particle core sizes (mean diameter $\langle d\rangle=9.7 \mathrm{~nm}$ ). They differ only in the additional biocompatible dextran layer (hydrodynamical size).

The magnetic interaction between the particles with diameters on the order of the mean value is too weak to provide their agglomeration into any heterogeneous 


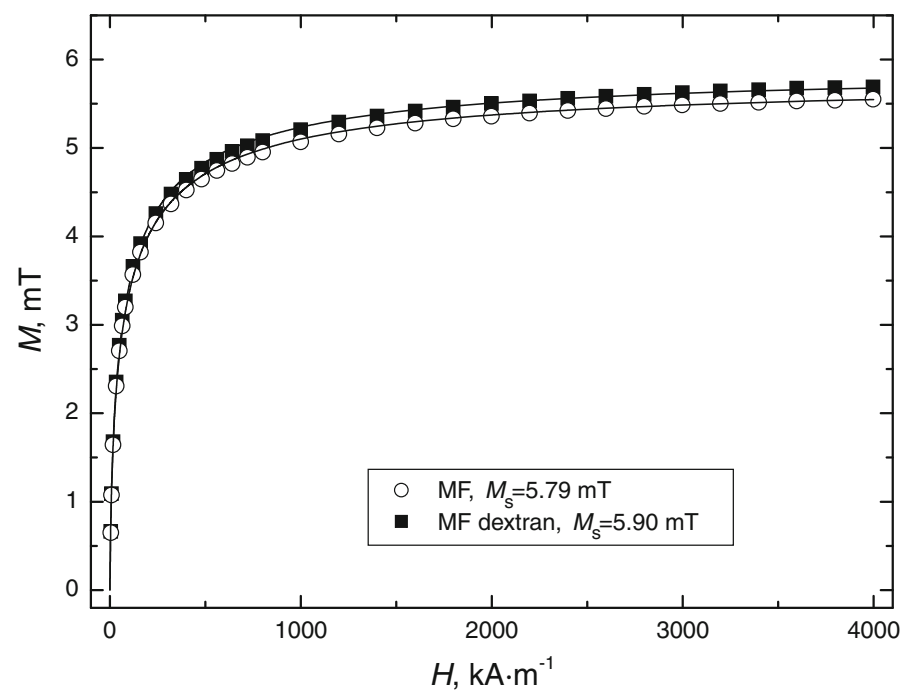

Fig. 3 Magnetization curves $M(H)$ obtained from SQUID for both samples studied

Table 1 Concentration of magnetic material, $\phi_{\mathrm{m}}$, saturation magnetization, $M_{\mathrm{S}}$, mean diameter of magnetic core, $\langle d\rangle$, and standard deviation of magnetic core size, $\sigma$, for studied samples

\begin{tabular}{lllll}
\hline Samples & $\phi_{\mathrm{m}}(\%)$ & $M_{\mathrm{S}}(\mathrm{mT})$ & $\langle d\rangle(\mathrm{nm})$ & $\sigma(\mathrm{nm})$ \\
\hline MF & 1.38 & 5.79 & 9.72 & 6.60 \\
MF dextran & 1.40 & 5.90 & 9.65 & 6.32 \\
\hline
\end{tabular}
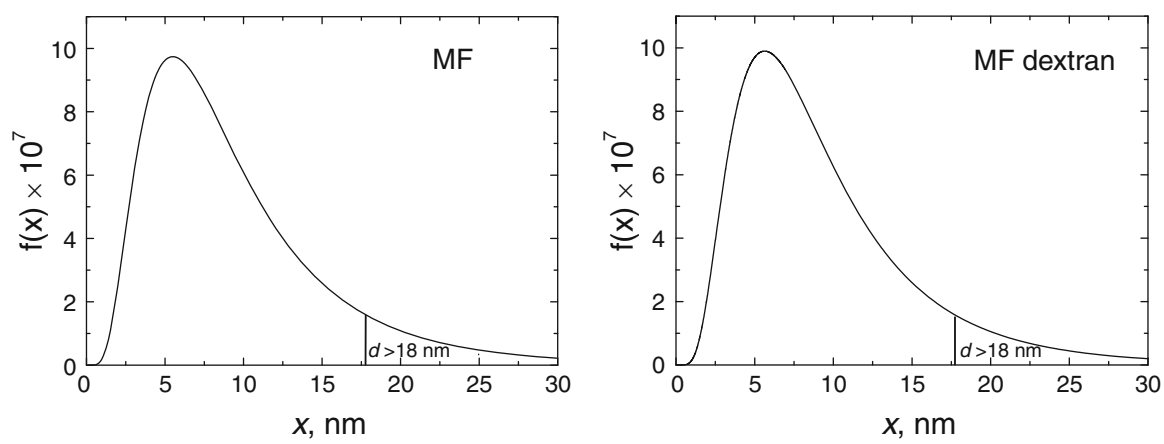

Fig. 4 Particle magnetic core size distributions calculated from SQUID data

aggregates. However, large particles with diameters about $16 \mathrm{~nm}$ to $18 \mathrm{~nm}$ (and larger) corresponding to the tails of the particle size distribution are able to condense into such structures [6]. Given the particle size distribution function $f(x)$, one can calculate the volume concentration of the large particles $(d>18 \mathrm{~nm})$ from the following relation: 


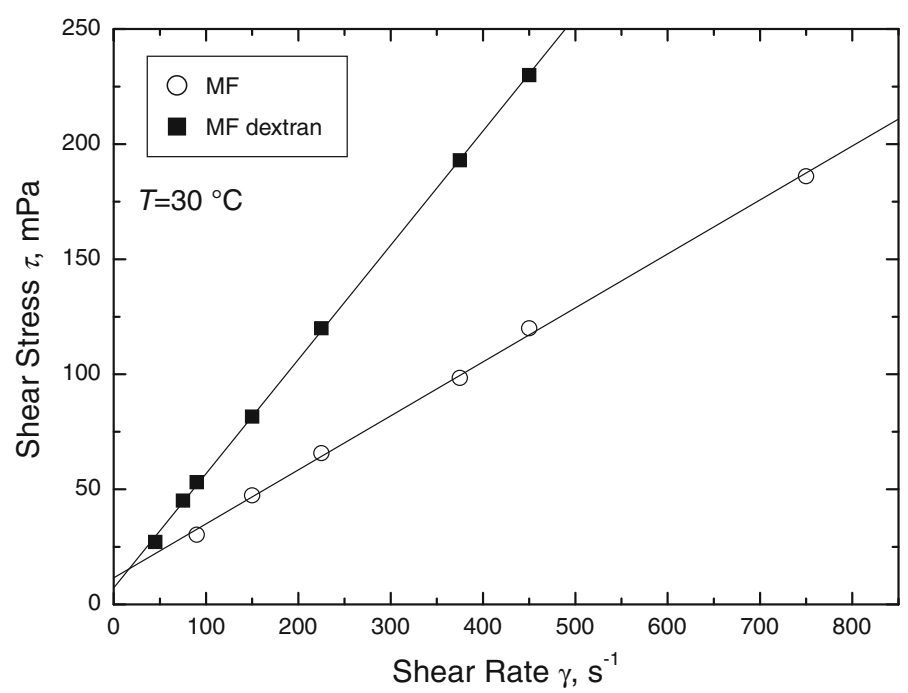

Fig. 5 Effect of the addition of dextran layer on shear stress versus shear rate flow curves

$$
\phi_{18}=\phi_{\mathrm{m}} \int_{18}^{\infty} f(x) \mathrm{d} x \text {. }
$$

For the magnetic fluid denoted as MF, $\phi_{18}=0.132 \%$, and for the fluid MF dextran, $\phi_{18}=0.125 \%$.

\section{Rheological Properties}

Rheological properties of the studied liquids in the absence of an external magnetic field were measured using a rotation Digital Brookfield Rheometer DV II+ in a coneplate geometry. Figure 5 shows the flow curves, i.e., the shear stress as a function of the shear rate. The effect of the addition of the biocompatible dextran layer is clearly visible. However, for both samples the flow curves are linear within the shear rate range of measurement $\left(20 \mathrm{~s}^{-1}\right.$ to $\left.750 \mathrm{~s}^{-1}\right)$. It means that in the absence of a magnetic field both ferrofluids studied exhibit Newtonian behavior. The measured viscosity of the magnetic fluids before and after the addition of dextran is plotted against the temperature in Fig. 6. In the absence of the magnetic field, the viscosity increases after the addition of dextran and decreases with temperature according to the Arrhenius equation $\left(\eta=9 \times 10^{-5} \exp \left(3123 T^{-1}\right)\right.$ for the MF and $\eta=2 \times 10^{-5} \exp \left(3905 T^{-1}\right)$ for the MF dextran, where $\eta$ is the dynamic viscosity [in $\mathrm{mPa} \cdot \mathrm{s}$ ] and $T$ is the temperature [in $\mathrm{K}]$ ).

The rheological measurements under the influence of the magnetic field were carried out using a rotational rheometer, Physica MCR300 (Anton Paar), equipped with a magnetorheological device, MRD180. It is a coaxial parallel plate-plate system with a diameter of $20 \mathrm{~mm}$ (PP 20/MRF). The distance between the plates was adjusted to 


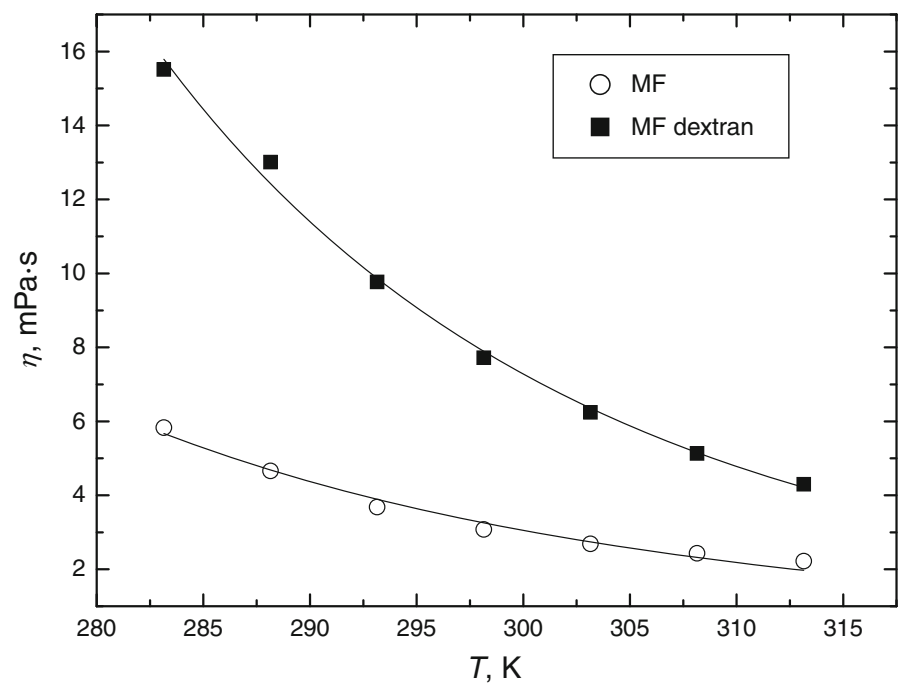

Fig. 6 Temperature dependence of the shear viscosity for MF and MF dextran magnetic fluids

$0.5 \mathrm{~mm}$. The built-in coil of the magnetorheological cell generated a magnetic field, $H$, that could be varied from 0 to $160 \mathrm{kA} \cdot \mathrm{m}^{-1}$. During the measurement the field was oriented perpendicular to the plates of the rheometer, and thus, perpendicular to the direction of the flow. The MR cell also included a water-based thermostatic system, so that a temperature of $20^{\circ} \mathrm{C}$ was maintained during all measurements. The MCR300 software then computed the shear stress versus shear rate flow curves for five values of the magnetic field intensity, $H=(0,30,60,90,160) \mathrm{kA} \cdot \mathrm{m}^{-1}$.

The measured viscosities for both samples in the field of $H=160 \mathrm{kA} \cdot \mathrm{m}^{-1}$ and without the presence of magnetic field are illustrated in Fig. 7. It can be seen that the magnetic field influences the viscosity of both samples; however, the effect is small in comparison with our previous study in transformer oil-based magnetic fluids [12]. The viscosity of magnetic fluids is determined by the interaction between molecules of the carrier liquid and between nanoparticles and the magnetic field which orients the magnetic particles along the direction of the field. The magnetic particles are rearranged under the applied magnetic field, and this leads to the formation of orderly microstructures and to the increase of the viscosity of magnetic fluids under the applied magnetic field. However, in our low-concentration samples, the distance between magnetic nanoparticles is large and so their interaction is weak. Therefore, the effect of the applied magnetic field on the magnetic fluids viscosity is rather small. It should be also noted that the magnitude of this effect is approximately the same for both of our samples.

In order to describe the rheological properties of magnetic fluids, the Bingham and Herschel-Bulkley models are usually used [14]. These are the generalized rheological models for viscoplastic flow with a yield stress. Both models describe the relation between the shear stress $\tau$, and the shear rate $\dot{\gamma}$, which has the following general form:

$$
\tau=\tau_{0}+k \dot{\gamma}^{n}
$$




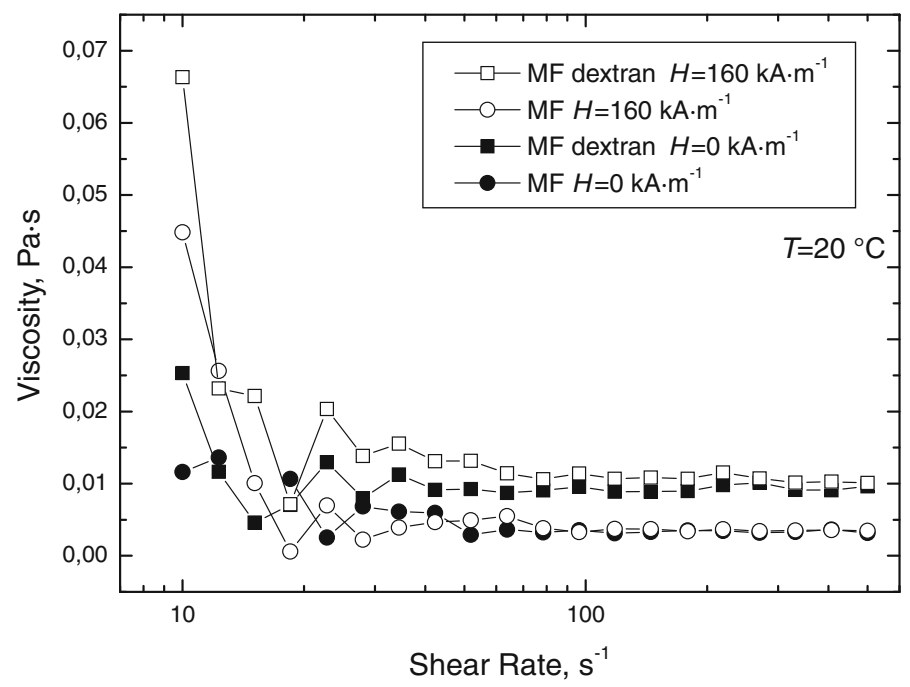

Fig. 7 Shear viscosity versus shear rate with and without magnetic field

where $\tau_{0}$ is the yield stress, $k$ is the consistency index, and $n$ is the power law index. When $\tau<\tau_{0}$, the material remains rigid; otherwise, the material flows either according to the power law showing shear thinning or shear thickening behavior $(n \neq 1)$ in the Herschel-Bulkley model, or according to the linear function in Bingham fluids $(n=1)$ [14]. Our preliminary calculations showed that $n \approx 1.0 \pm 0.1$ for all the samples studied. Thus, the results can be described by Bingham model flow curves which are shown in Fig. 8. The yield stress values obtained by fitting the model parameters to the experimental data are presented in Table 2. Within experimental error of the measured shear stress, the increasing trend of the yield stress is clearly visible in the range of 0 to $160 \mathrm{kA} \cdot \mathrm{m}^{-1}$ of the magnetic field strength. The rheological measurements also show that the addition of the biocompatible dextran layer leads to an increase of the yield stress.

Zubarev and Iskakova [6] considered a single domain, one-shell ferrofluid, filling a flat gap, placed into a perpendicular magnetic field. They arrived at the conclusion that the phase separation of the system into dense and dilute phases leads to the formation of a thermodynamically stable system of discrete domains, linking the gap walls. The domain density $\Phi$ can be found from the usual condition of the particles conservation,

$$
\Phi=\frac{\phi_{18}-\phi_{\mathrm{e}}}{\phi_{\mathrm{d}}-\phi_{\mathrm{e}}}
$$

where $\phi_{\mathrm{d}}$ and $\phi_{\mathrm{e}}$ are hydrodynamical volume concentrations of particles in the condensed (domains) and diluted phases, respectively. It was shown [6] that these domains are weakly dependent on the magnetic field strength and are typically of $\phi_{\mathrm{d}} \approx 0.5$ and $\phi_{\mathrm{e}} \approx 10^{-8}$. In external magnetic field when the domains span the walls of the gap, the ferrofluid exhibits quasielastic properties with respect to the strain (stress) in the 

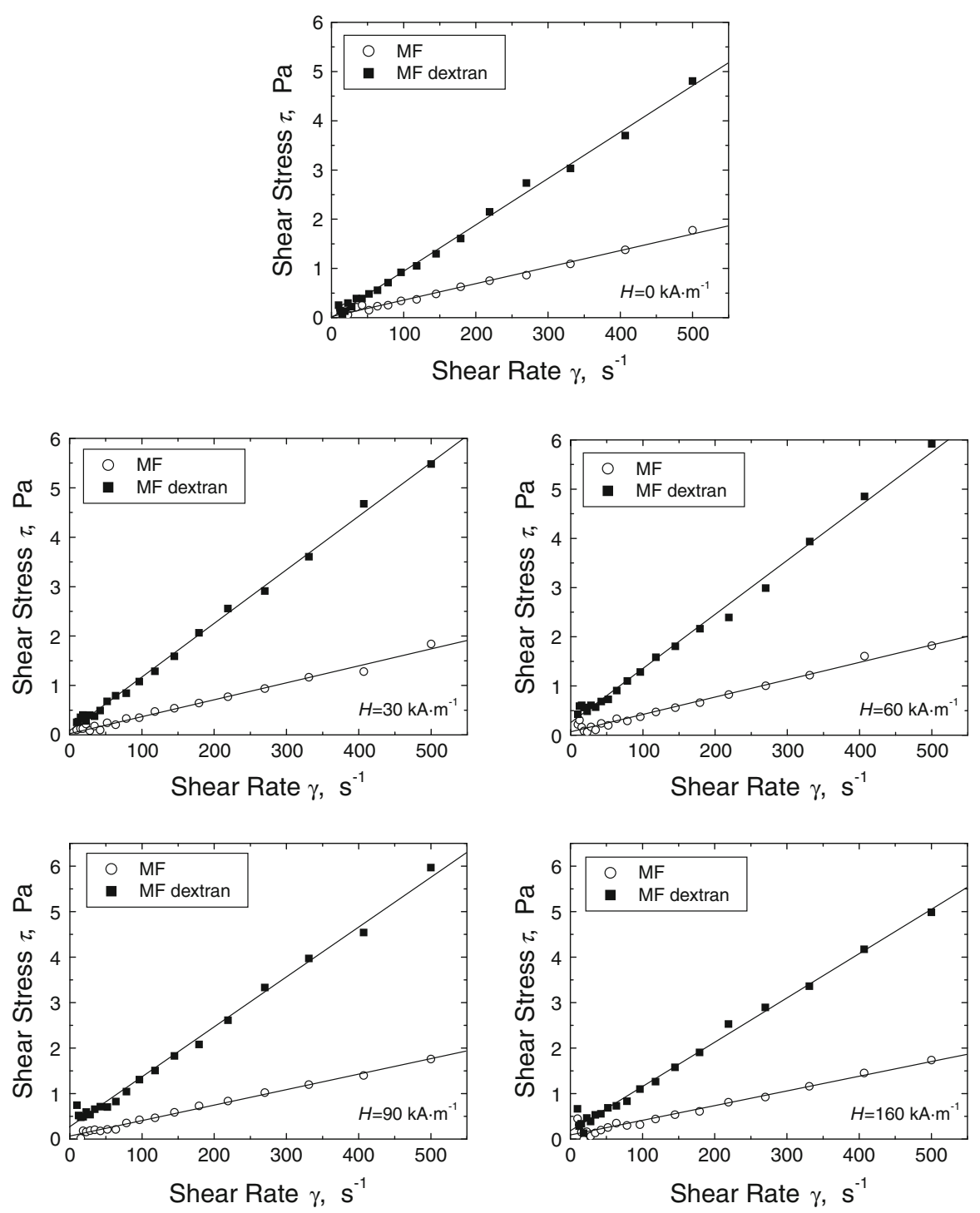

Fig. 8 Shear stress versus shear rate for different values of the magnetic field strength (including no-field curves). Solid lines present the Bingham rheological model fitted to the experimental data of samples with a sodium oleate stabilizing layer and with the additional biocompatible dextran layer

direction of the gap plane. When the stress exceeds a certain magnitude (yield stress), the quasielastic behavior of the ferrofluid transforms into the flow regime of the fluid.

In their analysis of rheological properties of magnetic fluids, Zubarev and Iskakova [6] distinguished between two microscopical mechanisms of the yield stress effect. The first one is connected with the fact that the elastic stress of the domain system as a function of the shear strain has a maximum equal to 
Table 2 Parameters of Bingham rheological model obtained by fitting the model to the shear stress versus shear rate experimental flow curves

\begin{tabular}{llllll}
\hline$H\left(\mathrm{kA} \cdot \mathrm{m}^{-1}\right)$ & $\mathrm{MF}$ & & \multicolumn{2}{l}{ MF dextran } \\
\cline { 2 - 3 } & $\tau_{0}(\mathrm{~Pa})$ & $k(\mathrm{~s})$ & & $\tau_{0}(\mathrm{~Pa})$ & $k(\mathrm{~s})$ \\
\hline 0 & $0.020 \pm 0.022$ & $0.0034 \pm 0.0002$ & & $0.004 \pm 0.020$ & $0.0094 \pm 0.0002$ \\
30 & $0.023 \pm 0.025$ & $0.0034 \pm 0.0002$ & & $0.085 \pm 0.025$ & $0.0108 \pm 0.0002$ \\
60 & $0.070 \pm 0.022$ & $0.0035 \pm 0.0002$ & & $0.250 \pm 0.039$ & $0.0109 \pm 0.0002$ \\
90 & $0.067 \pm 0.015$ & $0.0034 \pm 0.0001$ & & $0.274 \pm 0.042$ & $0.0042 \pm 0.0002$ \\
160 & $0.092 \pm 0.033$ & $0.0032 \pm 0.0002$ & & $0.183 \pm 0.039$ & $0.0098 \pm 0.0002$ \\
\hline
\end{tabular}

$$
\tau_{0}^{\mathrm{sat}}=0.162 \mu_{0} \phi_{\mathrm{md}}^{2} M_{\mathrm{b}}^{2} \Phi,
$$

where

$$
\phi_{\mathrm{md}}=\frac{\phi_{\mathrm{d}}}{\left(1+2 s / d_{\mathrm{m}}\right)^{2}},
$$

$M_{\mathrm{b}}=330 \mathrm{kA} \cdot \mathrm{m}^{-1}$ is the bulk magnetization of magnetite prepared by the coprecipitation method [15], $d_{\mathrm{m}}$ is the diameter of the magnetic core of the particle, and $s=2.5 \mathrm{~nm}$ is the thickness of the surfactant layer on the particle. The hydrodynamical diameter of the particle is $d=d_{\mathrm{m}}+2 s$. The concentration of the domains in the case of our magnetic fluids can be calculated by taking $\phi_{18}$ to be the concentration of the relatively large particles with diameters above $18 \mathrm{~nm}$, which are able to condense into domains. In case of our one-shell samples (with only a sodium oleate stabilizing layer), it leads to the value of $\Phi=0.00215$. Using these estimates, one obtains for the saturated value of the yield stress, $\tau_{0}^{\text {sat }}=1.25 \mathrm{~Pa}$.

The second mechanism of the yield stress in magnetic fluids distinguished by Zubarev and Iskakova is connected with the destruction of the "bridges" under the shear strain exceeding some critical value, $\gamma_{c}$, into separate drops, which do not link the gap walls. This mechanism should be applicable to the gaps with a thickness of about $1 \mathrm{~mm}$ and more. Because the drops do not span the gap, destruction of these domains leads to the beginning of the ferrofluid shear flow under the applied shear stress. The yield stress calculated according to this mechanism is given by the equation,

$$
\tau_{0}=\frac{1}{2} \mu_{0} H_{0}^{2} \frac{\gamma_{\mathrm{c}}}{\left(1+\gamma_{\mathrm{c}}^{2}\right)^{2}} \Phi
$$

From Fig. 8 in Ref. [6], one estimates the value $\gamma_{\mathrm{c}}=0.12$ for $H_{0}=160 \mathrm{kA} \cdot \mathrm{m}^{-1}$. This leads to the value of the yield stress $\tau_{0}=5.30 \mathrm{~Pa}$. The above calculations were performed for the samples with sodium oleate used as a stabilizing layer for which the thickness is better defined in comparison with samples possessing two layers. According to Zubarev and Iskakova [6], one can expect the experimentally measured yield stress to be the minimal of the stress, $\tau_{0}^{\text {sat }}$, determined in Eq. 4, and $\tau_{0}$ in Eq. 5. 
In the case of our MF samples, theory predicts the yield stress to attain a saturation value of about $1.25 \mathrm{~Pa}$. However, the yield stress measured for $H=160 \mathrm{kA} \cdot \mathrm{m}^{-1}$ is equal to $0.092 \mathrm{~Pa}$, which is about 12 times smaller than that predicted by the theory of Zubarev and Iskakova. The reason for that discrepancy, according to the authors of the theory, may lie in the slipping condition appearing in the experiment, that is, the slipping of the domains on the channel of flow, or in the way the yield stress is determined form flow curves by extrapolation of the shear stress results from the developed fluid regime to the point of zero shear rate [6].

\section{Conclusions}

The effect of a dextran additional layer on the rheological properties of a magnetic fluid was studied. The surface modification of magnetic nanoparticles with dextran enhances the stability of biocompatible magnetic fluids and leads to an increase of the viscosity of the magnetic fluids. A magnetic field can enhance the interaction among magnetic particles, and as a result, the viscosity increases under the external magnetic field. The magnetic field could also rearrange the magnetic particles leading to the formation of orderly microstructures and the appearance of elastic properties of the magnetic fluids as well as the yield stress effects. Our measurements show that the elastic properties of our systems can be qualitatively explained by the mechanisms predicted by Zubarev and Iskakova. However, quantitative estimation gives a yield stress about 12 times higher than its value obtained in the experiment.

Acknowledgments This work was supported by a Polish National Science Centre Grant No. DEC2011/03/B/ST7/00194, and by the Human Capital Operational Programme, Grant No. UDA-POKL.04.01. 01-00-133/09-00.

Open Access This article is distributed under the terms of the Creative Commons Attribution License which permits any use, distribution, and reproduction in any medium, provided the original author(s) and the source are credited.

\section{References}

1. R.W. Rosensweig, Ferrohydrodynamics (Cambridge University Press, Cambridge, 1985)

2. M.C. Bautista, O. Bomati-Miguel, M.P. Morales, C.J. Serna, S. Veintemillas-Verdaguer, J. Magn. Magn. Mater. 293, 20 (2005)

3. M. Tang, H. Dou, K. Sun, Polymer 47, 728 (2006)

4. J.W. Goodwin, Colloids and Interfaces with Surfactants and Polymers (Wiley, Chichester, 2009)

5. V. Socoliuc, D. Bica, L. Vekas, Magnetohydrodynamics 47, 201 (2011)

6. A.Y. Zubarev, L.Y. Iskakova, Physica A 365, 265 (2006)

7. V. Socoliuc, L. Vekas, Prog. Colloid Polym. Sci. 117, 131 (2001)

8. H. Shahnazian, D. Graf, D.Y. Borin, S. Odenbach, J. Phys. D: Appl. Phys. 42, 205004 (2009)

9. A. Mertelj, A. Rešetč, S. Gyergyek, D. Makovec, M. Čopič, Soft Matter 7, 125 (2011)

10. M. Koneracká, A. Antošová, V. Závišová, G. Lancz, Z. Gažová, K. Šipošová, A. Juríková, K. Csach, J. Kováč, N. Tomašovicová, M. Fabián, P. Kopčanský, Acta Phys. Pol. A 118, 983 (2010)

11. L. Dạbek, T. Hornowski, A. Józefczak, A. Skumiel, Arch. Acoust. 38, 93 (2013)

12. Z. Rozynek, A. Józefczak, K.D. Knudsen, A. Skumiel, T. Hornowski, O. Fossum, M. Timko, P. Kopčanský, M. Koneracká, Eur. Phys. J. E 34, 28 (2011) 
13. A. Józefczak, T. Hornowski, A. Skumiel, M. Łabowski, M. Timko, P. Kopčanský, M. Koneracká, A. Szlaferek, W. Kowalski, J. Magn. Magn. Mater. 321, 1505 (2009)

14. R.Y. Hong, J.H. Li, J.M. Qu, L.L. Chen, H.Z. Li, Chem. Eng. J. 150, 572 (2009)

15. M. Timko, A. Džarová, P. Kopčanský, V. Závišová, M. Koneracká, J. Kováč, A. Sprincová, M. Vaclavíková, L. Ivaničová, I. Vávra, Acta Phys. Pol. A 113, 573 (2008) 